\title{
Discovering structural motifs using a structural alphabet: Application to magnesium-binding sites Minko Dudev ${ }^{1}$ and Carmay Lim*1,2
}

Address: ${ }^{1}$ Institute of Biomedical Sciences, Academia Sinica, Taipei 115, Taiwan and 2Department of Chemistry, National Tsing-Hua University, Hsinchu 300, Taiwan

Email: Minko Dudev - frater_ia@yahoo.com; Carmay Lim* - carmay@gate.sinica.edu.tw

* Corresponding author

Published: 28 March 2007

BMC Bioinformatics 2007, 8:106 doi:10.1186/147|-2105-8-106
Received: 27 October 2006

Accepted: 28 March 2007

This article is available from: http://www.biomedcentral.com/I47/-2/05/8//06

(c) 2007 Dudev and Lim; licensee BioMed Central Ltd.

This is an Open Access article distributed under the terms of the Creative Commons Attribution License (http://creativecommons.org/licenses/by/2.0), which permits unrestricted use, distribution, and reproduction in any medium, provided the original work is properly cited.

\begin{abstract}
Background: For many metalloproteins, sequence motifs characteristic of metal-binding sites have not been found or are so short that they would not be expected to be metal-specific. Striking examples of such metalloproteins are those containing $\mathrm{Mg}^{2+}$, one of the most versatile metal cofactors in cellular biochemistry. Even when $\mathrm{Mg}^{2+}$-proteins share insufficient sequence homology to identify $\mathrm{Mg}^{2+}$-specific sequence motifs, they may still share similarity in the $\mathrm{Mg}^{2+}$-binding site structure. However, no structural motifs characteristic of $\mathrm{Mg}^{2+}$-binding sites have been reported. Thus, our aims are (i) to develop a general method for discovering structural patterns/motifs characteristic of ligand-binding sites, given the 3D protein structures, and (ii) to apply it to $\mathrm{Mg}^{2+}$ proteins sharing $<30 \%$ sequence identity. Our motif discovery method employs structural alphabet encoding to convert 3D structures to the corresponding ID structural letter sequences, where the $\mathrm{Mg}^{2+}$-structural motifs are identified as recurring structural patterns.
\end{abstract}

Results: The structural alphabet-based motif discovery method has revealed the structural preference of $\mathrm{Mg}^{2+}$-binding sites for certain local/secondary structures: compared to all residues in the $\mathrm{Mg}^{2+}$-proteins, both first and second-shell $\mathrm{Mg}^{2+}$-ligands prefer loops to helices. Even when the $\mathrm{Mg}^{2+}$-proteins share no significant sequence homology, some of them share a similar $\mathrm{Mg}^{2+}$-binding site structure: $4 \mathrm{Mg}^{2+}$-structural motifs, comprising $21 \%$ of the binding sites, were found. In particular, one of the $\mathrm{Mg}^{2+}$-structural motifs found maps to a specific functional group, namely, hydrolases. Furthermore, 2 of the motifs were not found in non metalloproteins or in $\mathrm{Ca}^{2+}$-binding proteins. The structural motifs discovered thus capture some essential biochemical and/or evolutionary properties, and hence may be useful for discovering proteins where $\mathrm{Mg}^{2+}$ plays an important biological role.

Conclusion: The structural motif discovery method presented herein is general and can be applied to any set of proteins with known 3D structures. This new method is timely considering the increasing number of structures for proteins with unknown function that are being solved from structural genomics incentives. For such proteins, which share no significant sequence homology to proteins of known function, the presence of a structural motif that maps to a specific protein function in the structure would suggest likely active/binding sites and a particular biological function. 


\section{Background}

Magnesium is one of the most versatile metal cofactors in cellular biochemistry, serving both intra and extracellular, catalytic and/or structural roles [1]. It is used to stabilize a variety of protein structures; e.g., the interface of the ribonucleotide reductase subunits [2]. It is also used to stabilize nucleic acids by alleviating electrostatic repulsion between negatively charged phosphates. Furthermore, $\mathrm{Mg}^{2+}$, together with $\mathrm{Ca}^{2+}$, stabilize biological membranes by charge neutralization after binding to the carboxylated and phosphorylated headgroups of lipids. It also activates enzymes that regulate the biochemistry of nucleic acids such as restriction nucleases, ligases, and topoisomerases, and is essential for the fidelity of DNA replication [1]. Divalent $\mathrm{Mg}^{2+}$ is a "hard" ion and prefers "hard" ligands of low polarizability like oxygen. It tends to bind directly to the amino acid residues, primarily to the Asp/Glu carboxylic side chains, followed by the Asn/Gln side chains or peptide backbone carbonyl groups [3]. The rest of the metal coordination sphere, which is usually octahedral, is complemented by water ligand(s).

Unlike $\mathrm{Zn}^{2+}$ and $\mathrm{Ca}^{2+}$-binding sites, only a few, relatively short, sequence motifs have been discovered for $\mathrm{Mg}^{2+}$ proteins with close sequence homology. These include the NADFDGD- motif, found in different RNA polymerases, DNA Pol I and HIV reverse transcriptase, and the -YXDDor -LXDD- motifs in reverse transcriptase and telomerase, where residues in bold are the $\mathrm{Mg}^{2+}$ ligands [4]. As the known $\mathrm{Mg}^{2+}$ sequence motifs are short, they could easily be found in other non $\mathrm{Mg}^{2+}$-proteins and would not be expected to be $\mathrm{Mg}^{2+}$-specific. Interestingly, some homology in the 3D structure of the $\mathrm{Mg}^{2+}$-binding sites has been observed for certain classes of enzymes such as restriction enzymes, bacterial and viral RNase $\mathrm{H}$ domains, and viral integrases [4]. However, systematic studies of the structural preference/conservation of $\mathrm{Mg}^{2+}$-binding sites in nonhomologous proteins have not been reported; hence, no structural motifs of the $\mathrm{Mg}^{2+}$-binding sites have been extracted.

The aims in this work are to address the following intriguing questions: (1) Do $\mathrm{Mg}^{2+}$-binding sites exhibit any preference for certain local/secondary structures? If so, which types of local/secondary structures are favored and which are disfavored? (2) Even when the $\mathrm{Mg}^{2+}$-proteins share no significant sequence homology, do they share a similar $\mathrm{Mg}^{2+}$-binding site structure? (3) If structural motifs of the $\mathrm{Mg}^{2+-b i n d i n g}$ sites exist, do they map to specific protein functions? (4) Are the structural motifs $\mathrm{Mg}^{2+}$-specific? In particular, are they found in proteins that do not bind metal ions or in proteins that bind $\mathrm{Ca}^{2+}$, which like $\mathrm{Mg}^{2+}$, is also a divalent "hard" ion, binding preferentially to "hard" oxygen-containing ligands?
To address the aforementioned questions, we have developed a general strategy for discovering 3D motifs that are hidden in the local structure of the active/binding site, based on the fact that the local structure is generally more evolutionary conserved than the amino acid sequence [5]. The 3D motifs of the metal-binding sites were obtained by encoding the $3 \mathrm{D}$ representation based on Cartesian coordinates into a $1 \mathrm{D}$ representation based on a 16-letter structural alphabet $[6,7]$. The structural alphabet represents recurring short structural prototypes and has been used to (i) compare/analyze 3D structures [8-10], (ii) predict protein $3 \mathrm{D}$ structures from amino acid sequences $[6,11]$, (iii) reconstruct the protein backbone [12], and (iv) model loops [13]. However, it has not been used to discover structural motifs of metal/ligand-binding sites in proteins. First, the structural-alphabet based motif discovery approach was validated by using it to "rediscover" the structural motif of $\mathrm{Cys}_{4}$ Zn-finger domains, which are known to adopt a specific structure. Next, it was used to discover structural motifs of $\mathrm{Mg}^{2+}$-binding sites in a set of nonredundant $\mathrm{Mg}^{2+}$-proteins sharing $<30 \%$ sequence identity. The results reveal clear trends in the structural composition of $\mathrm{Mg}^{2+}$-binding sites, $4 \mathrm{Mg}^{2+}$-structural motifs, and important relationships between these motifs and other features of the proteins. The specificity of the structural motifs discovered for certain $\mathrm{Mg}^{2+}$-binding sites was assessed by determining their occurrence in a set of nonredundant non-metal containing protein structures and in a set of nonredundant $\mathrm{Ca}^{2+}$-bound protein structures.

\section{Results}

\section{Validation against Proteins with known Structural Motifs}

To test the structural alphabet-based strategy for discovering metal-binding site structural motifs, a database of 42 structural zinc sites from 29 proteins in previous work [14] was searched for proteins containing the $\mathrm{C}(2) \mathrm{C}(13-$ $15) \mathrm{C}(2) \mathrm{C}$ sequence motif, where the number in parentheses indicates the number of amino acid residues separating the conserved Zn-binding cysteines. Proteins with such a sequence motif belong to the Zn-finger family of the nuclear receptor type, having a $\mathrm{Cys}_{4} \mathrm{Zn}$-binding site [15], which is known to adopt a specific structure. Each of the Zn-proteins containing the $\mathbf{C}(2) \mathbf{C}(13-15) \mathbf{C}(2) \mathbf{C}$ sequence motif was represented by a $1 \mathrm{D}$ structural alphabet, as described in Methods and illustrated in Figure 1. All of these proteins were found to possess a $f(2) o(13-$ 15) $f(2) m$ structural motif of the Zn-binding site (see Figure 1). This shows that the structural-alphabet based approach for discovering new structural motifs seems promising.

\section{Structural Preference of $\mathbf{M g}^{\mathbf{2}+\text { Binding Sites }}$}

Although the $70 \mathrm{Mg}^{2+}$-proteins used herein share $<30 \%$ sequence identity, do their $\mathrm{Mg}^{2+}$-binding sites prefer cer- 


\section{$>$ 1HCQ Chain A}

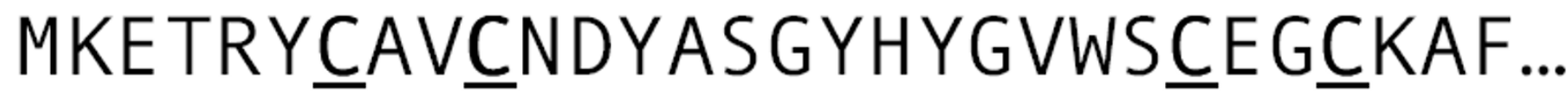

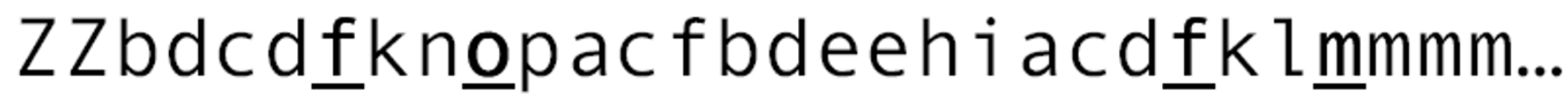
$>1$ LAT Chain A

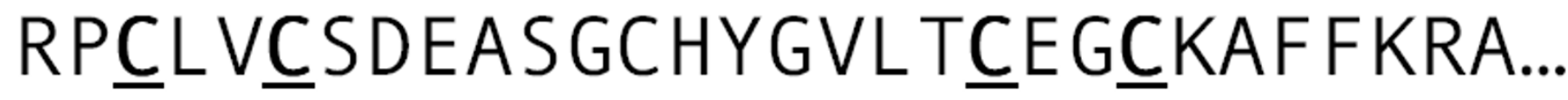

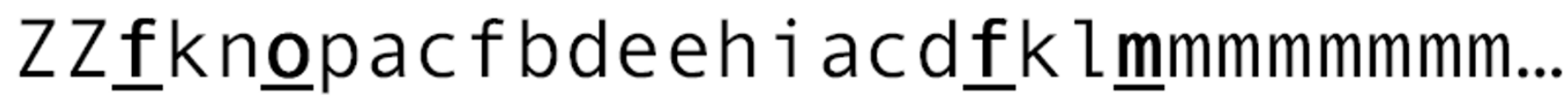
$>2 N L L$ Chain B DELㄷVㄷGDKATGYHYRC ITㅌGㅡKGFFRR...

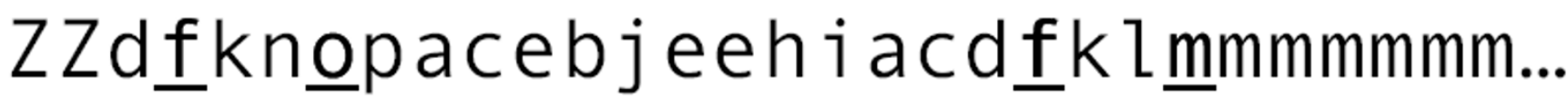

\section{Figure I}

$\mathbf{Z n}$-binding site structural motifs derived from the structural alphabet representation of $3 \mathbf{Z n}$-finger proteins. For each protein, the PDB entry and chain is given, followed below by its amino acid sequence (in capital letters), aligned with the corresponding structural alphabet representation (lower-case letters); ' $Z$ ', means a letter cannot be assigned to this residue (see Methods). $\mathrm{Zn}^{2+}$-binding residues are underlined and in bold. Only the first 30 amino acid residues are shown. The $C_{\alpha}$ root-mean-square deviation RMSD of ILAT and 2NLL from IHCQ are 1.73 and I.33 $\AA$, respectively, whereas that of ILAT from $2 N L L$ is $1.25 \AA$.

tain local structures? To answer this question, the 3D structure of each of the 70 nonredundant $\mathrm{Mg}^{2+}$ proteins was represented by a 16-letter structural alphabet (see Methods and Additional file 1), and the frequencies of the letters in all the first-and second-shells as well as in the entire $\mathrm{Mg}^{2+}$ dataset were compared (see Figure 2). The results reveal a clear preference towards certain types of local structures in the $\mathrm{Mg}^{2+}$-binding sites. The ' $b$ ', ' $d$ ', ' $f$ ', and ' $h$ ' frequencies of first-shell $\mathrm{Mg}^{2+}$-ligands and the ' $d$ ', ' $e$ ', ' $f$ ' and ' $k$ ' frequencies of second-shell $\mathrm{Mg}^{2+-}$ ligands are statistically significantly higher than the respective frequencies of all the amino acid residues in the dataset (see Table 1). Both first and second-shell $\mathrm{Mg}^{2+}$-ligands favor the ' $d$ ' and ' $f$ ' structures. Furthermore, the first-shell (but not the second-shell) $\mathrm{Mg}^{2+}$-ligands strongly prefer the local structure ' $h$ ', whose frequency of first-shell ligands is 5.3fold greater than that of all residues in $\mathrm{Mg}^{2+}$ proteins. However, compared to all amino acid residues in the $\mathrm{Mg}^{2+}$ proteins, both first and second-shell $\mathrm{Mg}^{2+}$-ligands disfavor certain local protein structures such as the ' $c$ ' and ' $m$ ' structures: The ' $c$ ', ' $i$ ', ' $m$ ', and ' $p$ ' frequencies of first-shell $\mathrm{Mg}^{2+}$-ligands and the ' $a$ ', ' $c$ ', ' $m$ ' and ' $o$ ' frequencies of second-shell $\mathrm{Mg}^{2+}$-ligands are statistically significantly lower than the respective frequencies of all the amino acid residues in the dataset (see Table 1).

To relate the observed bias of the first-shell Mg2+-ligands for certain structures to standard regular and irregular secondary structures, the percentage frequency distribution of first-shell, second-shell, and all amino acid residues that are found in $\alpha$-helices, $\beta$-strands, or loops in the $\mathrm{Mg} 2+$-proteins according to the secondary structure information in the Protein Data Bank [16] (PDB) files were computed (see Figure 3). The loop occurrence frequency of the first or second-shell $\mathrm{Mg} 2+$-residues $(47-50 \%)$ is significantly higher than that of all residues ( $32 \%)$ with pvalues $\leq 0.014$ (see Table 1 ). However the $\beta$-sheet occurrence frequency of the first or second-shell $\mathrm{Mg} 2+$-residues $(\sim 29 \%)$ is not significantly higher than that of all residues $(\sim 22 \%)$. In contrast, the $\alpha$-helix occurrence frequency of 
Table I: The letter and secondary structural element (SSE) frequency distributions and 2-sample T-tests of first-and second-shell amino acid residues vs. all amino acid residues in the $\mathbf{M g}^{2+}$-proteins

\begin{tabular}{|c|c|c|c|c|c|c|}
\hline \multirow[b]{2}{*}{ Letter, $x^{\mathrm{a}}$} & \multicolumn{3}{|c|}{ |st-shell vs. all residues } & \multicolumn{3}{|c|}{$2^{\text {nd }}$-shell vs. all residues } \\
\hline & $v_{x, l} / v_{x, \text { all }}^{b}$ & T-test ${ }^{c}$ & P-value ${ }^{c, d}$ & $v_{x, 2} / v_{x, \text { all }} \mathrm{e}^{\mathrm{e}}$ & T-test ${ }^{c}$ & $P$-value ${ }^{c, d}$ \\
\hline$a$ & 1.47 & 1.4037 & 0.0802 & 0.57 & 2.4731 & 0.0067 \\
\hline$b$ & 1.86 & 2.7909 & 0.0027 & 1.20 & 1.2200 & 0.1113 \\
\hline c & 0.56 & 2.0160 & 0.0219 & 0.50 & 4.3510 & $<0.0001$ \\
\hline$d$ & 1.23 & 1.7376 & 0.0412 & 1.23 & 3.1829 & 0.0008 \\
\hline e & 1.46 & 1.0111 & 0.1560 & 2.03 & 4.1825 & $<0.0001$ \\
\hline$f$ & 1.47 & 1.9389 & 0.0263 & 1.70 & 5.4060 & $<0.0001$ \\
\hline$g$ & 1.15 & 0.2494 & 0.4015 & 1.18 & 0.5381 & 0.2953 \\
\hline$h$ & 5.29 & 9.3752 & $<0.0001$ & 1.19 & 0.7921 & 0.2142 \\
\hline$i$ & 0 & 1.8928 & 0.0292 & 1.34 & 1.1910 & 0.1168 \\
\hline$j$ & 2.21 & 1.6156 & 0.0531 & 1.54 & 1.3401 & 0.0901 \\
\hline$k$ & 1.40 & 1.4992 & 0.0669 & 1.60 & 4.1820 & $<0.0001$ \\
\hline 1 & 0.76 & 0.9209 & 0.1786 & 1.08 & 0.5978 & 0.275 \\
\hline$m$ & 0.52 & 2.9377 & 0.0017 & 0.74 & 5.2192 & $<0.0001$ \\
\hline$n$ & 0.53 & 1.1306 & 0.1291 & 0.88 & 0.5208 & 0.3013 \\
\hline 0 & 1.52 & 1.4066 & 0.0798 & 0.35 & 3.3637 & 0.0004 \\
\hline$p$ & 0 & 3.1174 & 0.0009 & 0.77 & 1.3204 & 0.0934 \\
\hline \multicolumn{7}{|l|}{ SSE, $x$} \\
\hline Loop & 1.56 & 2.5575 & 0.0053 & 1.47 & 2.1874 & 0.0144 \\
\hline$\beta$-strands & 1.30 & 1.0780 & 0.1405 & 1.34 & 1.2170 & 0.1118 \\
\hline$\alpha$-helices & 0.47 & 3.6454 & 0.0002 & 0.51 & 3.3621 & 0.0004 \\
\hline
\end{tabular}

a |6-letter structural alphabet defined by de Brevern and co-workers (see Methods and original reference) [6]. 'The ratio of the letter/SSE ' $x$ ' frequency of first-shell amino acid residues to that of all amino acid residues in the $70 \mathrm{Mg}^{2+}$ proteins. CThe statistical analyses were carried out using the package, SAS/STAT version 8 (SAS Institute, NC). dP-values $<0.05$ are highlighted in bold. eThe ratio of the letter/SSE ' $x$ ' frequency of secondshell amino acid residues to that of all amino acid residues in the $70 \mathrm{Mg}^{2+}$ proteins.

the first or second shell Mg2+-residues (22-23\%) is nearly half of the respective frequency of all residues $(\sim 46 \%)$ with p-values $\leq 0.0004$.

In summary, the $\mathrm{Mg}^{2+-}$-binding sites generally prefer certain local structures: compared to all amino acid residues in the $\mathrm{Mg}^{2+}$ proteins, both first and second-shell ligands tend to prefer loops to helices. This may be due to the need to position not only the first and second-shell ligands, but also the helix dipole, in a proper orientation for metal binding.

\section{Structural Motifs of $\mathbf{M g}^{2+}-$ Binding Sites}

Even when the $\mathrm{Mg}^{2+}$-proteins share no significant sequence homology ( $<30 \%$ sequence identity), do any of them share a common structure of the metal-binding site? Such structural motifs are defined in this work to exist if 3 or more $\mathrm{Mg}^{2+}$-binding sites have the same first-shell letters and similar interletter spacing (see Methods and Additional file 1). These structural motifs are listed in Table 2 and illustrated in Figure 4, while first-shell structural patterns that are common to only $2 \mathrm{Mg}^{2+}$-binding sites are listed in Additional file 2. For the first shell, 4 structural motifs, representing about a fifth $(16 / 77$ or $21 \%)$ of all $\mathrm{Mg}^{2+}$-binding sites, were discovered. All 4 motifs occur in proteins whose functions are either $\mathrm{Mg}^{2+}$-dependent or whose native co-factors are $\mathrm{Mg}^{2+}$ according to UniProt and/or the literature. Consistent with the above finding that the ' $h$ ' structure is preferred by the first-shell $\mathrm{Mg}^{2+}$-ligands, it is in the middle of all 4 motifs and the partial motif ' $f(1-2) h$ ' accounts for half of the $\mathrm{Mg}^{2+}$-proteins with structural motifs. For the second shell, too many residues define the $\mathrm{Mg}^{2+}$-binding site; hence not enough $\mathrm{Mg}^{2+}$ binding sites possess the same second-shell letters and similar interletter spacing. However, 5 partial motifs for the second shell were found: These are $f(1) l m, k l(0-1) m$, $d(1-2) f f, d(1) e(1) i(0-5) l, f(1) l(18-25) d$, with an occurrence frequency of $21,12,11,8$, and $6 \%$, respectively.

Each of the 4 motifs in Table 2 is found in proteins containing Mg2+-binding domains belonging to the same superfamily. This is evidenced by the fact that proteins with the same $\mathrm{Mg} 2+$-structural motif have $\mathrm{Mg} 2+$-binding domains belonging to the same superfamily with the same CATH numbers (Table 2), implying structurally homologous domains. For example, all 3 proteins with the $\mathrm{f}(2) \mathrm{h}(126-158) \mathrm{m}$ motif possess in common a Mg2+binding domain belonging to the fructose-1,6-bisphosphatase, subunit A, domain 1 superfamily (CATH number 3.30.540.10). Likewise, all 5 proteins with the $\mathrm{k}(26-$ 29)h(1)a motif possess Mg2+-binding domains with the same CATH number, 3.40.50.970. The fact that the motifs 


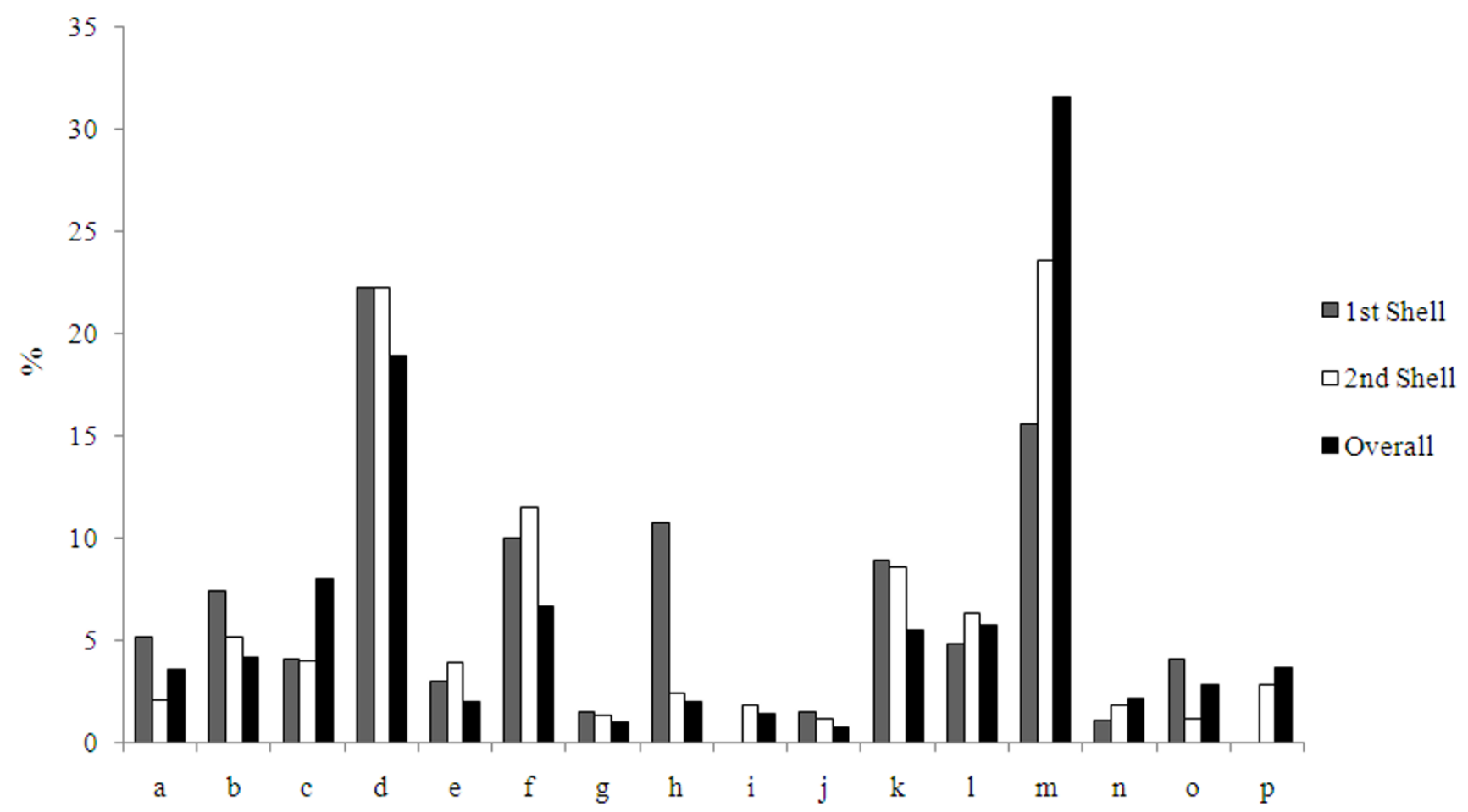

Figure 2

The percentage letter frequency distributions of first-shell amino acid residues (gray), second-shell amino acid residues (white), and all amino acid residues (black) in the $\mathbf{M g}^{\mathbf{2 +}}$-proteins. There is a total of 25,406 amino acid residues in the $\mathrm{Mg}^{2+}$-proteins, of which 250 are in the first shell, while 898 are in the second shell

are found in structurally homologous $\mathrm{Mg} 2+$-binding domains further supports the use of the structural alphabet to discover motifs.

The first-shell motifs discovered herein can also help to uncover relationships between proteins with unassigned CATH numbers. For example, 2 of the 3 proteins with the $e(24-47) h(24) k$ motif (1SJC and 1TKK) possess $\mathrm{Mg}^{2+}$ binding domains pertaining to the enolase superfamily (CATH number 3.20.20.120), whereas the third protein (2AKZ) has not yet been assigned a domain and therefore has no CATH number. Although the n-acylamino acid racemase (1SJC) and gamma enolase (2AKZ) proteins do not share significant sequence homology (only 15.4\% identity) and overall structure similarity (protein backbone rmsd = $17.5 \AA$ ), they possess similar $\mathrm{Mg}^{2+}$-binding site structures (backbone rmsd of the first-shell letters = $0.5 \AA$ ), as shown in Figure 5. In analogy, 3 of the 5 proteins with the $f(1) h(109-349) b$ motif (1008, 1WPG, and 2B82) possess $\mathrm{Mg}^{2+-b i n d i n g}$ domains with the same CATH number (3.40.50.1000), whereas the other 2 proteins (1U7P and $2 \mathrm{C} 4 \mathrm{~N}$ ) have not yet been chopped into domains and therefore have not been assigned CATH numbers. The results in Table 2 predict that the $\mathrm{Mg}^{2+}$ dependent phosphatase (1U7P) and NagD (2C4N) pro- teins are likely to possess $\mathrm{Mg}^{2+}$-binding domains that are structurally homologous to those assigned with the CATH number 3.40.50.1000.

\section{Relation between $\mathrm{Mg}^{2+}$-Structural Motifs and PROSITE Sequence Motifs}

To see if any of the $\mathrm{Mg}^{2+}$-proteins containing structural motifs match sequence motifs stored in the PROSITE database [17], the sequences of the proteins listed in Table 2 were scanned for the occurrence of PROSITE sequence motifs. None of the proteins match any PROSITE sequence motifs encompassing residues involved in $\mathrm{Mg}^{2+}$ binding. However, the halotolerance protein hal 2 (1KA1) containing the $f(2) h(126-158) m$ motif matched 2 inositol monophosphatase family signatures (PROSITE PDOC00547) containing conserved metal-binding residues. This supports the ' $f(2) h(126-158) m$ ' motif as a signature of $\mathrm{Mg}^{2+}$-binding sites.

\section{Relation between $\mathbf{M g}^{2+}$-Structural Motifs and Protein Function}

Do any of the structural motifs found for the $\mathrm{Mg}^{2+}$-proteins map to specific protein functions? To answer this question, for each of the $\mathrm{Mg}^{2+}$-proteins found with a structural motif, the functional group of the protein from the 


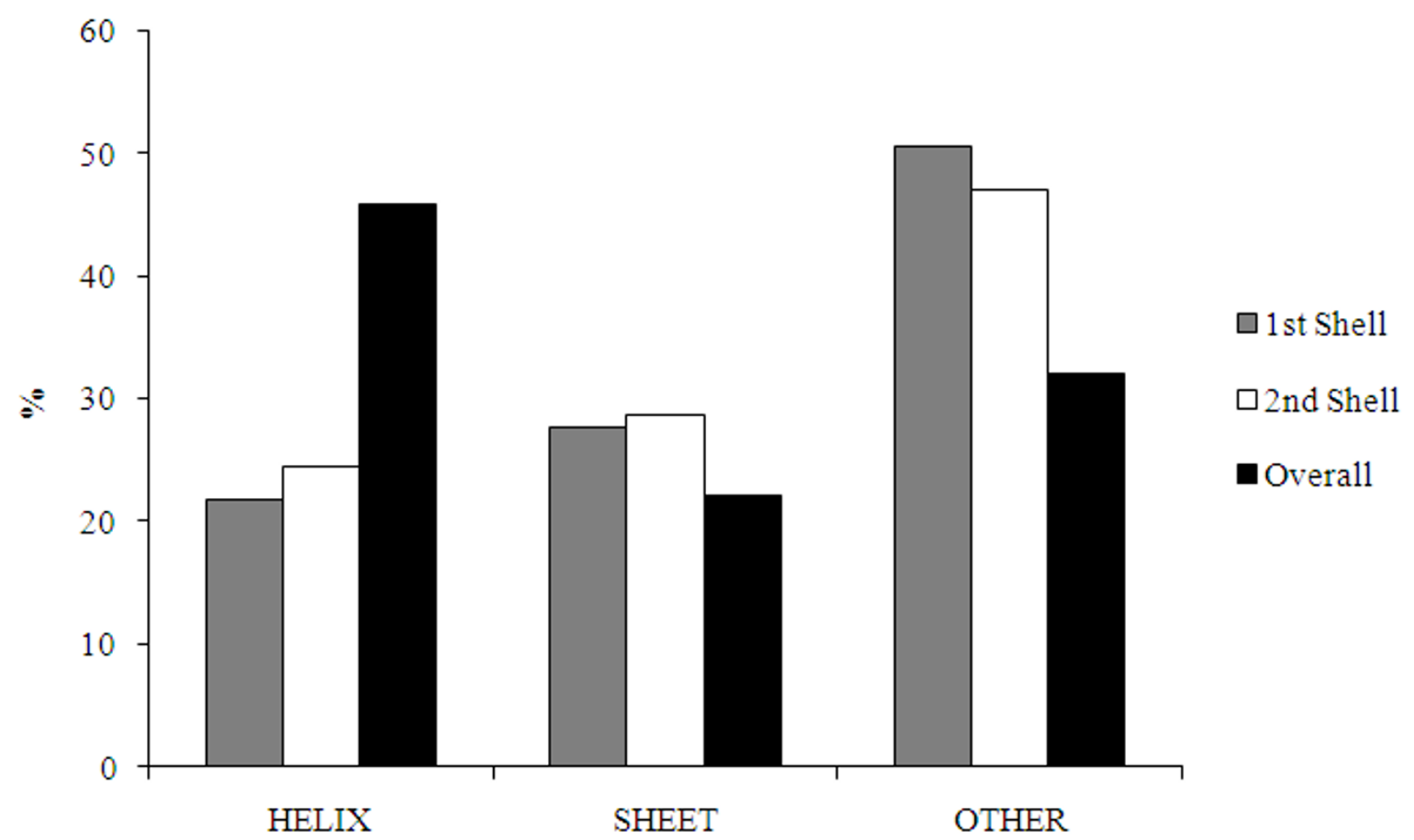

Figure 3

The percentage secondary structure frequency distributions of first-shell amino acid residues (gray), secondshell amino acid residues (white), and all amino acid residues (black) in the $\mathbf{M g}^{2+}$-proteins. The amino acid residues found in $\alpha$-helices, $\beta$-strands, or loops are according to the secondary structure information in the PDB files.

PDB header and enzyme classification (EC) code, if applicable, are listed in Table 2. Note that proteins belonging to the same functional group have the same first EC number. The results in Table 2 show that most of the structural motifs found for the $\mathrm{Mg}^{2+}$-proteins map to certain protein functions. For example, proteins with the partial $f(1-2) h$ motif are all hydrolases, catalyzing the hydrolytic cleavage of mostly ester bonds (EC3.1.-.-), except for beta-phosphoglucomutase (1008), which is an isomerase converting beta-D-glucose 1-phosphate to betaD-glucose 6-phosphate. Interestingly, although class b acid phosphatase (2B82) and the halotolerance protein hal 2 (1KA1) contain structurally nonhomologous $\mathrm{Mg}^{2+}$ binding domains with different CATH numbers, both are phosphoric monoester hydrolases (EC3.1.3.-). Proteins with the $e(24-47) h(24) k$ motif are either lyases and/or isomerases, whereas proteins with the $k(26-29) h(1) a$ motif have even more diverse functions: 3 are oxidoreductases (1POX, 1UMD, 2C3M), one is a lyase (1ZPD) and the other is a transferase (1ITZ). This shows that even if the proteins share structurally homologous domains (CATH number 3.40.50.970) and structurally similar
$\mathrm{Mg}^{2+}$-binding sites, as represented by the $k(26-29) h(1) a$ motif, they can perform different functions.

\section{Statistical Significance of the $\mathbf{M g}^{\mathbf{2 +}}$-Structural Motifs}

Do the structural motifs found for $\mathrm{Mg}^{2+}$-proteins in Table 2 occur in other proteins that do not bind metal ions? To address this question, de Brevern's databank of protein structures that have been encoded into 1D structural sequences was searched for the occurrence of each of the 4 structural motifs listed in Table 2. Consistent with the $\mathrm{Mg}^{2+}$ and $\mathrm{Ca}^{2+}$ datasets, proteins in de Brevern's databank sharing $\geq 30 \%$ sequence identity with $\geq 2.5$ - $\AA$ resolution $\mathrm{X}$-ray structures were removed. Proteins in de Brevern's databank whose structures are complexed with metal ions were also removed, yielding a set of 385 non-homologous test proteins. In order to eliminate those matched structural letters that cannot spatially bind $\mathrm{Mg}^{2+}$, the maximum $\mathrm{C}_{\alpha}-\mathrm{C}_{\alpha}$ distance between any pair of $\mathrm{Mg}^{2+-}$-ligands belonging to proteins of a given structural motif in Table 2 was extracted; this distance is $9.32 \AA$ for the $e(24-$ $47) h(24) k$ motif, $8.32 \AA$ for $f(1) h(109-349) b, 8.44 \AA$ for $f(2) h(126-158) m$, and $7.86 \AA$ for $k(26-29) h(1) a$. For a given structural motif in Table 2, matched letters in the 
Table 2: Ist-shell structural motifs in $\mathrm{Mg}^{2+}$-proteins

\begin{tabular}{|c|c|c|c|c|c|}
\hline Motifa & PDB code & $\mathrm{Mg}^{2+}$-Ligands & CATH numberb & Functional Groupc & $E C$ code $^{d}$ \\
\hline \multirow[t]{3}{*}{$e(24-47) h(24) k$} & ISJC & $D^{189}, E^{214}, D^{239}$ & 3.20 .20 .120 & Lyase $^{e}$, Isomerasef & - \\
\hline & ITKK & $D^{191}, E^{219}, D^{244}$ & 3.20 .20 .120 & Isomerase ${ }^{f}$ & - \\
\hline & $2 A K Z$ & $D^{244}, E^{292}, D^{317}$ & - & Lyase $^{e}$ & 4.2.1.11 \\
\hline \multirow[t]{5}{*}{$f(I) h(109-349) b$} & 1008 & $D^{1008}, D^{1010}, D^{1170}$ & 3.40 .50 .1000 & Isomerase $\mathrm{f}^{\mathrm{f}}$ & 5.4.2.6 \\
\hline & IU7P & $D^{11}, D^{13}, D^{123}$ & NYC & Hydrolaseg & - \\
\hline & IWPG & $D^{351}, T^{353}, D^{703}$ & 3.40 .50 .1000 & Hydrolaseg & 3.6.3.8 \\
\hline & $2 \mathrm{~B} 82$ & $D^{44}, D^{46}, D^{167}$ & 3.40 .50 .1000 & Hydrolaseg & 3.1.3.2 \\
\hline & $2 \mathrm{C} 4 \mathrm{~N}$ & $D^{9}, D^{11}, D^{201}$ & NYC & Hydrolaseg & - \\
\hline \multirow{3}{*}{$f(2) h(\mid 26-158) m$} & $|K A|$ & $D^{142}, D^{145}, D^{294}$ & 3.30 .540 .10 & Hydrolaseg & 3.1.3.7 \\
\hline & INUY & $D^{1118}, D^{1121}, E^{1280}$ & $\begin{array}{l}3.30 .540 .10+ \\
3.40 .190 .80\end{array}$ & Hydrolaseg & 3.1 .3 .11 \\
\hline & $2 \mathrm{BJI}$ & $E^{1090}, D^{1093}, D^{1220}$ & $\begin{array}{l}3.30 .540 .10+ \\
3.40 .190 .80\end{array}$ & Hydrolaseg & 3.1.3.25 \\
\hline \multirow[t]{5}{*}{$\mathrm{k}(26-29) \mathrm{h}(\mathrm{I}) \mathrm{a}$} & IITZ & $D^{168}, N^{198},\left.\right|^{200}$ & 3.40 .50 .970 & Transferase $^{h}$ & 2.2.1.1 \\
\hline & IPOX & $\mathrm{D}^{447}, \mathrm{~N}^{474}, \mathrm{Q}^{476}$ & $\begin{array}{l}3.40 .50 .970+ \\
3.40 .50 .1220\end{array}$ & Oxidoreductase ${ }^{i}$ & I.2.3.3 \\
\hline & IUMD & $\mathrm{D}^{175}, \mathrm{~N}^{204}, \mathrm{Y}^{206}$ & 3.40 .50 .970 & Oxidoreductase ${ }^{i}$ & I.2.4.4 \\
\hline & IZPD & $\mathrm{D}^{440}, \mathrm{~N}^{467}, \mathrm{G}^{469}$ & 3.40 .50 .970 & Lyase $^{\mathrm{e}}$ & 4.I.I.I \\
\hline & $2 C 3 M$ & $\mathrm{D}^{963}, \mathrm{~T}^{991}, \mathrm{~V}^{993}$ & 3.40 .50 .970 & Oxidoreductase ${ }^{i}$ & 1.2.7.1 \\
\hline
\end{tabular}

aThe number in parentheses indicates the number of residues separating the letters corresponding to the $\mathrm{Mg}^{2+}$-bound residues. ${ }^{\text {bThe }} \mathrm{CATH}$ code of the domain containing the $\mathrm{Mg}^{2+}$-ligands; a dash implies that no domain could be assigned to the PDB entry, while NYC means the protein has not yet been chopped. CThe functional group from the PDB header. dThe enzyme class from PDBsum [25]; a dash means no EC code was found. eLyases (EC4---) catalyze $\mathrm{C}-\mathrm{C} / \mathrm{O} / \mathrm{N}$ and other bond cleavage; e.g., $\mathrm{RCOCOOH} \rightarrow \mathrm{RCOH}+\mathrm{CO}_{2}$. flsomerases (EC5---) catalyze geometric changes within a molecule. sHydrolases (EC3---) catalyze hydrolytic bond cleavage: $A B+\mathrm{H}_{2} \mathrm{O} \rightarrow \mathrm{AOH}+\mathrm{BH}$. ${ }^{\mathrm{h}}$ Transferases $(\mathrm{EC} 2---)$ catalyze $A B+\mathrm{C} \rightarrow \mathrm{A}+\mathrm{BC}$. iOxidoreductases (ECl---) catalyze oxido-reductions: $\mathrm{AH}+\mathrm{B} \rightarrow \mathrm{A}+\mathrm{BH}$ (reduced) and $\mathrm{A}+\mathrm{O} \rightarrow \mathrm{AO}$ (oxidized).

test proteins whose $\mathrm{C}_{\alpha}-\mathrm{C}_{\alpha}$ distances exceed the respective maximum distance were eliminated. This resulted in no matches for the $e(24-47) h(24) k$ and $f(2) h(126-158) m$ motifs, whereas 2 proteins (1C3K, 1GPE) contained the $f(1) h(109-349) b$ motif, and another 2 proteins (1A7U, 1JFR) contained the $k(26-29) h(1) a$ motif. A check of the literature confirmed that these 4 proteins $(1 \mathrm{C} 3 \mathrm{~K}, 1 \mathrm{GPE}$ 1A7U, 1JFR) do not bind metal ions. This suggests that (i) the $4 \mathrm{Mg}^{2+}$-structural motifs discovered are statistically significant, and (ii) the $e(24-47) h(24) k$ and $f(2) h(126-$ $158) \mathrm{m}$ motifs could be used to predict metal-binding sites.

\section{Metal Preference of the $\mathbf{M g}^{\mathbf{2 +}}$-Structural Motifs}

To check the specificity of the 4 structural motifs in Table 2 for $\mathrm{Mg}^{2+}$, the same procedure used to represent the $\mathrm{Mg}^{2+-b i n d i n g ~ s i t e s ~ i n ~ t e r m s ~ o f ~ t h e i r ~ l o c a l ~ s t r u c t u r e ~ w a s ~}$ repeated for $\mathrm{Ca}^{2+}$, which is the metal ion most similar to $\mathrm{Mg}^{2+}$. Both $\mathrm{Mg}^{2+}$ and $\mathrm{Ca}^{2+}$ are closed-shell divalent cations belonging to the same group (IIA) with similar chemical properties: They are both "hard" dications that prefer to bind directly to "hard" oxygen-containing anions, and are hence often found to bind in the same protein cavity [18]. Thus, the 3D structure of each of the 177 nonredundant $\mathrm{Ca}^{2+}$ proteins was represented by a 16-letter structural alphabet (see Methods), and the 1D structural letter rep- resentation of the $230 \mathrm{Ca}^{2+}$-binding sites are listed in Additional file 3.

None of the structural motifs in Table 2 or Additional file 2 were found in 3 or more $\mathrm{Ca}^{2+}$-binding sites, and therefore cannot be classified as $\mathrm{Ca}^{2+}$-structural motifs according to our definition. The $f(1) h(109-349) b$ motif is found in the $\mathrm{Ca}^{2+-b i n d i n g ~ s i t e ~ o f ~ t h e ~ h y d r o l a s e ~ f r o m ~ t h e ~}$ haloacid dehalogenase family (2FI1), which appears to utilize $\mathrm{Mg}^{2+}$ as a natural co-factor [19]. Although the $k(26-29) h(1) a$ motif is found in the calcium-binding sites of the transketolase protein (1TRK) and benzoylformate decarboxylase (1Q6Z), the latter is a $\mathrm{Mg}^{2+}$-dependent enzyme [20]. The $e(24-47) h(24) k$ and $f(2) h(126-158) m$ motifs did not match any first-shell structural letters of the $\mathrm{Ca}^{2+-b i n d i n g ~ s i t e s, ~ i n d i c a t i n g ~ t h a t ~ t h e y ~ s e e m ~ t o ~ f a v o r ~} \mathrm{Mg}^{2+}$ over its competitor, $\mathrm{Ca}^{2+}$.

\section{Discussion and conclusion Comparison with Previous Structural Motif Discovery Methods}

Assuming that similarity in the local active site structure implies similarity in biological function, 3D patterns/ templates of key active sites have been used to suggest the function of a protein whose structure is known. The 3D patterns/templates have been constructed either manually 

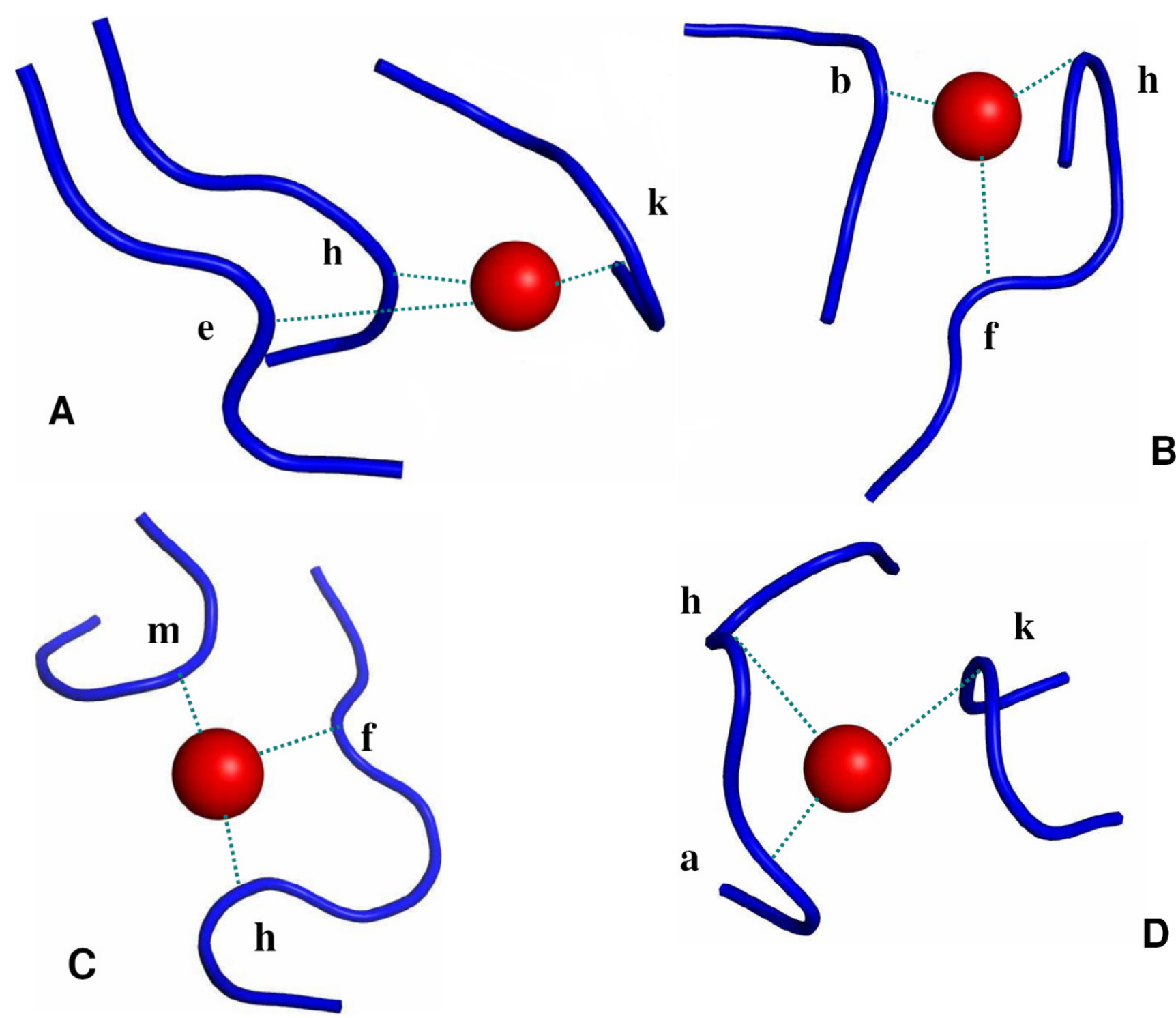

Figure 4

The conserved local structures of the $4 \mathbf{M g}^{2+}$-structural motifs. (a) e(24-47)h(24)k, (b) $f(I) h(I 09-349) b$, (c) $f(2) h(I 26-$ I58)m, and (d) k(26-29)h(I)a.

or automatically using various methods, which have been reviewed recently by Watson et al. [21]. Recently, 3D templates in the absence of experimental data have been constructed using the evolutionary trace method to identify evolutionarily important, solvent accessible residues that cluster in the protein structure [22]. Furthermore, structural motifs for the metal-binding sites of 3 distinct metalloenzymes families; viz., DNase 1 homologs, dimetallic phosphatases, and dioxygenases, have been obtained by first identifying physical chemical property-based sequence motifs in homologous protein sequences, and subsequently identifying those motifs whose structures are conserved in members of a family/superfamily
$[23,24]$. However, to the best of our knowledge, 3D patterns of key active sites and recurrent patterns (structural motifs) have not been identified using the structural alphabet to convert $3 \mathrm{D}$ structures to the respective $1 \mathrm{D}$ letter sequences. Also, systematic studies of the structural preference or conservation of $\mathrm{Mg}^{2+}$-binding sites in nonhomologous proteins and $\mathrm{Mg}^{2+}$-specific structural motifs have not been reported.

\section{Advantages of the Structural-Alphabet Based Motif Discovery Approach}

This work presents the first application of the structural alphabet approach to define the $3 \mathrm{D}$ patterns of metal 


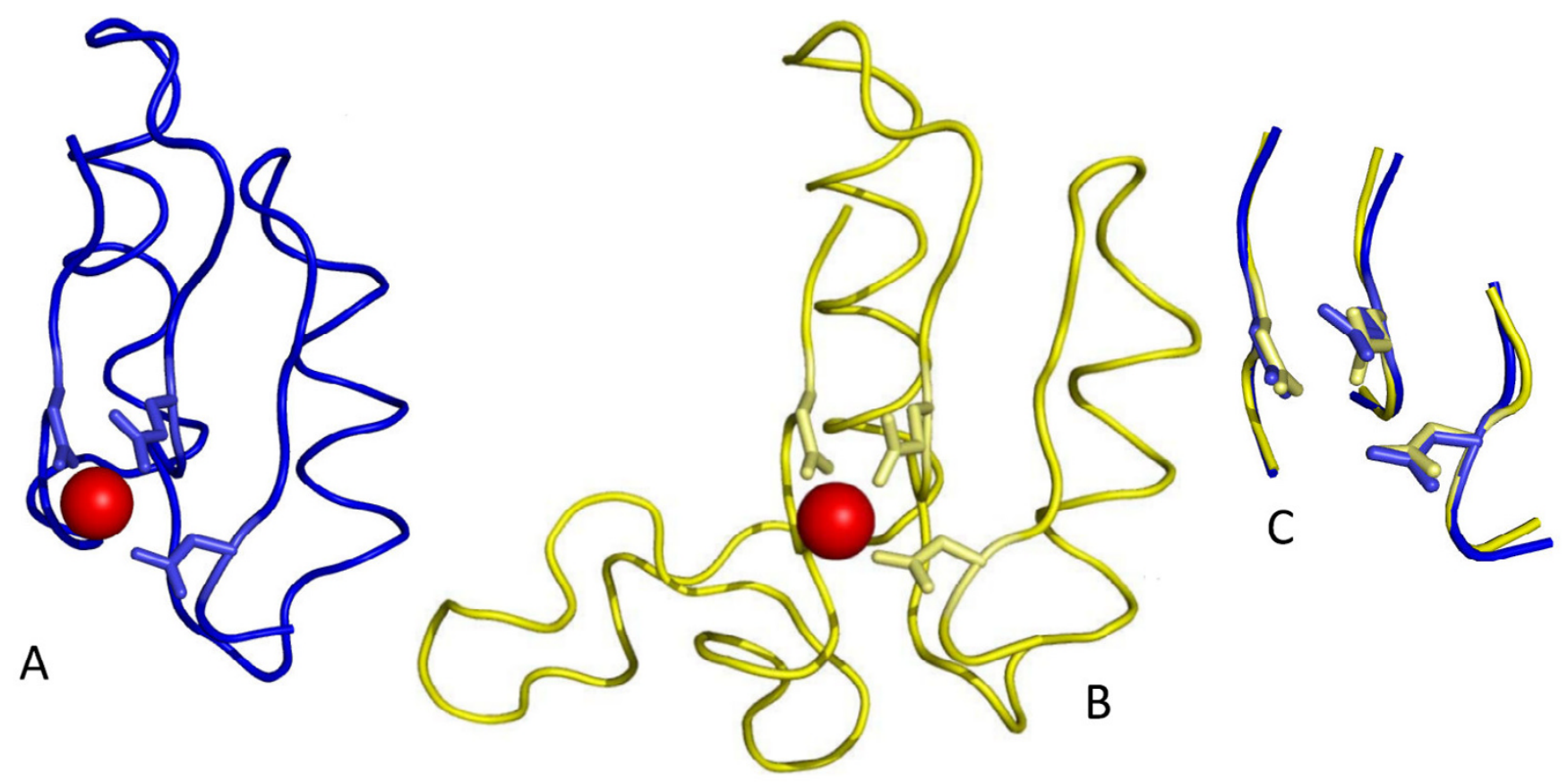

Figure 5

The conserved binding site of $\mathbf{2}$ nonhomologous $\mathbf{M g}^{2+}$-proteins. (a) Cartoon diagram of the metal-binding domain in $\mathrm{N}$-acylamino acid racemase (ISJC). (b) Cartoon diagram of the metal-binding domain in gamma enolase (2AKZ). (c) Superposition of the first-shell structural letters of ISJC (blue) and 2AKZ (yellow).

active sites and to identify recurrent patterns (structural motifs). The method requires as input only the $3 \mathrm{D}$ protein structure to define a 1D structural representation of the respective active site. The structural alphabet-based approach has several advantages: (i) It is efficient at handling many structures as it takes less than a minute on a present-day PC to convert a 3D structure to the corresponding $1 \mathrm{D}$ structural sequence. (ii) It requires no sequence comparisons, no parameters or scoring functions and can thus produce consistent structural motifs, whose structures are readily visualized, as illustrated in Figures 4 and 5. (iii) It is general and can be used to define $3 \mathrm{D}$ patterns not only in metal-binding sites, but also in enzyme active sites, ligand-binding clefts and interacting regions between proteins and their respective partners. The 3D patterns/motifs discovered could be incorporated in methods to detect metal/ligand-binding sites to improve their prediction accuracy.

\section{Secondary Structure Preference of $\mathbf{M g}^{\mathbf{2 +}-B i n d i n g ~ R e s i d u e s}$}

In this work, the structural alphabet-based approach has been used to reveal the structural preference of $\mathrm{Mg}^{2+}$-binding sites. Even though helix-like segments represented by the letter ' $\mathrm{m}$ ' is the most common building block of the $\mathrm{Mg}^{2+}$-proteins in the dataset, residues that bind $\mathrm{Mg}^{2+}$ disfavor helices, but favor loops. The similarity in the structural preference of the first and second-shell residues supports previous conclusions regarding the relationship between these 2 layers; namely, the structure and properties of the $2^{\text {nd- }}$ shell are dictated by those of the $1^{\text {st }}$ layer [14].

\section{Similar $\mathbf{M g}^{2+}$-Binding Site Structures in Dissimilar Protein Sequences}

The motif discovery method herein has revealed 4 structural motifs, comprising $21 \%$ of the $\mathrm{Mg}^{2+}$-binding sites. The 3D structural motifs discovered seems to have more predictive utility in identifying $\mathrm{Mg}^{2+}$-binding sites than 1D sequence motifs: A scan of the $\mathrm{Mg}^{2+}$-protein sequences in our dataset for the occurrence of sequence motifs stored in the PROSITE [17] database yielded only a single positive match, 1WC1, which contains a PROSITE sequence motif predicting the protein to bind $\mathrm{Mg}^{2+}$. However, the ScanProsite results did not identify any of the $\mathrm{Mg}^{2+}$ proteins with structural motifs.

\section{Functional Preference of the $\mathbf{M g}^{2+}-$ Structural Motifs}

The structural motifs discovered generally relate to the biological role of $\mathrm{Mg}^{2+}$ and the function of the respective proteins. They capture some essential biochemical and/or evolutionary properties, as proteins found to contain a specific structural motif possess structurally homologous $\mathrm{Mg}^{2+}$-binding domains, even though they share no significant sequence homology. Furthermore, the $f(2) h(126-$ 
158) $m$ motif maps to a specific functional group, namely, hydrolases, indicating the apparent importance of the local $\mathrm{Mg}^{2+}$-binding site structure for the function of these hydrolases. As the $f(2) h(126-158) m$ motif was not found in non-metalloproteins and in $\mathrm{Ca}^{2+}$-binding proteins, the presence of this motif in a novel protein structure may suggest a likely $\mathrm{Mg}^{2+}$-binding site and the protein function. On the other hand, the other 3 motifs map to more than one functional group, suggesting that the local $\mathrm{Mg}^{2+}$ binding site structure is not the only determinant of the protein's function.

\section{Why $\mathrm{Mg}^{2+}$ Specific Structural Motifs are Not Found For Most $\mathbf{M g}^{2+}$-Proteins}

Out of the 70 nonhomologous $\mathrm{Mg}^{2+}$-proteins, only 16 have first-shell structural motifs, while the rest do not seem to possess any metal-binding site structural motifswhy? One reason might be that some $\mathrm{Mg}^{2+}$-structural motifs may have been missed out in this work. As the dataset employed only proteins with $\mathrm{Mg}^{2+}$-bound structures (see Database subsection below), some PDB structures complexed with heavier metal ions such as $\mathrm{Mn}^{2+}$ may in fact correspond to native $\mathrm{Mg}^{2+}$-binding site(s); moreover, not all structures of proteins whose native cofactor is $\mathrm{Mg}^{2+}$ have been solved. A second reason is that for native $\mathrm{Mg}^{2+}$-binding sites that can accommodate other metal ions such as $\mathrm{Ca}^{2+}$ or $\mathrm{Mn}^{2+}$, the binding-site structure need not be conserved in order to recognize a specific metal co-factor. A third reason is that although $\mathrm{Mg}^{2+}$ occupied the binding site in the $3 \mathrm{D}$ structure, it is not the native cofactor. Although all 70 proteins are bound to $\mathrm{Mg}^{2+}$ in our dataset, according to PDBSUM [25] and from the UniProt annotation and references therein, 14 proteins do not employ $\mathrm{Mg}^{2+}$ as the native co-factor, while for 6 proteins, the native metal-cofactor is apparently not known (see Additional file 1). For example, calbindin d9K is a vitamin $\mathrm{D}$-dependent calcium-binding protein, but in the 1IG5 structure, the native cofactor $\mathrm{Ca}^{2+}$ has been replaced by $\mathrm{Mg}^{2+}$. In $\mathrm{Mg}^{2+}$-proteins with multiple $\mathrm{Mg}^{2+}$ binding sites, one or more sites may be non-native, as they have been artificially induced by the high $\mathrm{Mg}^{2+}$ concentration used during crystallization. In these cases, the local structure of the non-native metal-binding site would not be expected to share any similarity with that of a native $\mathrm{Mg}^{2+}$-binding site, where the conserved local structure (as in the $f(2) h(126-158) m$ motif) plays an important role in the protein's function. The absence of structural motifs for non-native $\mathrm{Mg}^{2+-b i n d i n g ~ s i t e s ~ i n d i r e c t l y ~ s u p p o r t s ~ o u r ~}$ strategy.

\section{Methods \\ Database}

A set of 70 nonredundant $\mathrm{Mg}^{2+}$ proteins was created by searching the PDB [16] for structures with resolution < $2.5-\AA$ containing $\mathrm{Mg}^{2+}$ with $<30 \%$ sequence identity.
Structures with residues missing in the middle of the sequence were excluded because such gaps in the structure could alter the spacing in the binding-site motifs (see below). Structures with $\mathrm{Mg}^{2+}$ bound to $<3$ protein ligands were also excluded, as 2-residue motifs cannot be considered specific enough for any practical use. The resulting $\mathrm{Mg}^{2+}$ dataset comprise 77 binding sites in 70 proteins. Note that although most $\mathrm{Mg}^{2+}$-proteins have only one binding site, some proteins have more than one $\mathrm{Mg}^{2+}$ binding sites (PDB entries 1MXG, 1NUY, 1VCL, 1WL6, $2 \mathrm{BJI}$, and 2BVC). A set of nonredundant $\mathrm{Ca}^{2+}$ proteins was created following the same procedure used to create the $\mathrm{Mg}^{2+}$ dataset. This resulted in $230 \mathrm{Ca}^{2+}$-binding sites in 177 proteins. The PDB entries, EC code, and amino acid residues bound to the metal ion in the $77 \mathrm{Mg}^{2+}$ and 230 $\mathrm{Ca}^{2+}$ sites are given in Additional files 1 and 3, respectively.

\section{The Structural Alphabet}

Each metalloprotein structure was encoded into its 1D structural sequence according to the original structural alphabet defined by de Brevern and co-workers [6]. We refer the reader to the original work [6] for details of how this alphabet was devised, and briefly outline the procedure here. The backbone of each protein from a nonredundant protein structure database was represented by consecutive 5-residue segments, each described by a vector of 8 backbone dihedral angles $V\left(\psi_{\mathrm{n}-2}, \phi_{\mathrm{n}-1}, \psi_{\mathrm{n}-1}, \phi_{\mathrm{n}^{\prime}} \psi_{\mathrm{n}^{\prime}}\right.$ $\left.\phi_{n+1}, \psi_{n+1}, \phi_{n+2}\right)$. The dissimilarity between 2 vectors $V_{1}$ and $V_{2}$ of dihedral angles is measured by the root-meansquare deviations of the dihedral angle values (rmsda), which is defined as the Euclidean distance among the 4 links:

$\operatorname{rmsda}\left(V_{1}, V_{2}\right)=\sqrt{\frac{\sum_{i=1}^{4}\left[\psi_{i}\left(\vec{V}_{1}\right)-\psi_{i}\left(\vec{V}_{2}\right)\right]^{2}+\left[\phi_{i+1}\left(\vec{V}_{1}\right)-\phi_{i+1}\left(\vec{V}_{2}\right)\right]^{2}}{8}}$

Using an unsupervised cluster analyzer based on the above rmsda of the segments, 16 letters (also called protein blocks) were identified, which in turn comprise the structural 'alphabet'.

\section{Converting 3D Structure to ID Structural Alphabet}

The $3 \mathrm{D}$ structures of the $70 \mathrm{Mg}^{2+}$ and $177 \mathrm{Ca}^{2+}$ proteins were converted into strings of structural letters using the program PBE published in ref. 9. For a given $n$ - residue protein, $n$ - 4 letter assignments were obtained by scanning the protein sequence using a 5-residue sliding window. The structure of each 5-residue segment is compared with that of each of the 16 letters and the letter that has the closest structure (as measured by the rmsda) to the 5-residue segment is assigned to the middle residue of that segment. This process is illustrated in Figure 6: The first letter is assigned to the $3^{\text {rd }}$ residue, Val, representing the first 5 - 


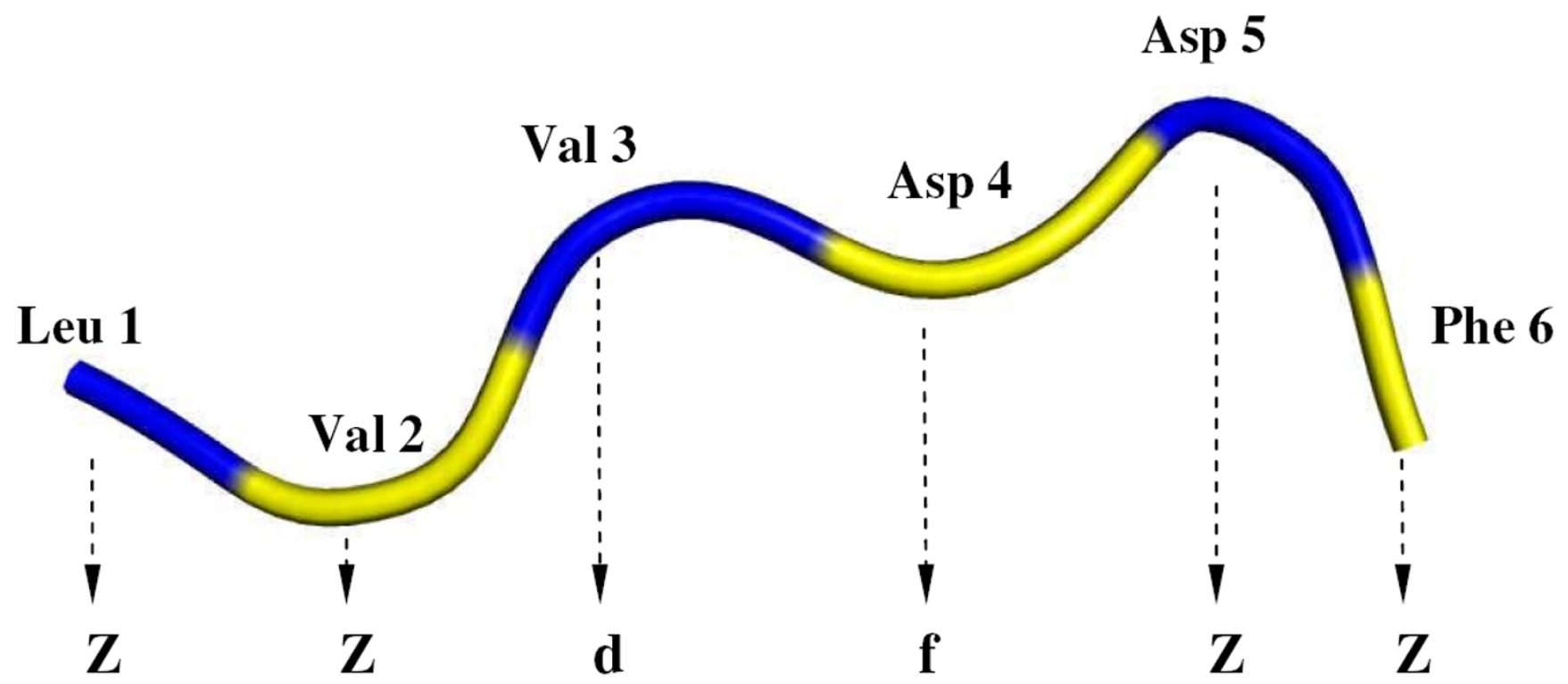

Figure 6

Conversion of the 3D protein backbone into a ID structural alphabet representation. The first 2 and the last 2 residues are assigned ' $Z$ ', meaning a letter cannot be assigned at these residues. The first valid assignment is ' $d$ ', at $V a l 3$ and spanning residues I to 5 . The next is assigned to Asp 4 and spans residues 2 to 6 .

residue segment. Its structure is closest to that of the structural letter ' $d$ ', therefore Val 3 is assigned ' $d$ '. Note that no letters can be assigned to the first 2 and last 2 residues of each protein.

\section{Definition of Ist and $2^{\text {nd }}$-Shell Metal Ligands}

Analyses of high-resolution X-ray structures with crystallographic $R$ factor $\leq 0.065$ of small metal complexes in the Cambridge Structural Database [26] have shown that the mean $1^{\text {st- }}$ shell $\mathrm{Mg}^{-\mathrm{O}_{\text {water }}} \mathrm{Mg} \mathrm{O}_{\text {carboxylate' }}$ and $\mathrm{Mg}-\mathrm{O}_{\text {alcohol }}$ distances do not exceed $2.11 \AA$, while the Ca-O $\mathrm{O}_{\text {water, }} \mathrm{Ca}$ $\mathrm{O}_{\text {carboxylate, }} \mathrm{Ca}-\mathrm{O}_{\text {alcohol}}$, and $\mathrm{Ca}-\mathrm{N}_{\text {imidazole }}$ bond distances do not exceed $2.55 \AA$ [27]. To account for the lower resolution of the PDB structures, a slightly larger cutoff was used to locate the $1^{\text {st }}$-shell amino acid ligands. Thus, the $\mathrm{Mg}^{2+}$ and $\mathrm{Ca}^{2+}$ ligands were defined as residues with a donor atom within $2.5 \AA$ and $2.7 \AA$ from the metal ion, respectively. The heavy atoms of the metal-bound amino acid residues were then selected as centers to search for the $2^{\text {nd }}$-shell protein ligands using a hydrogen-bonding cutoff of $3.5 \AA$ [28]. Note that water molecules in the first and second shells were not identified, as they were not used to define a structural motif.

\section{Definition of I $^{\text {st }}$ and $2^{\text {nd }}$-Shell Structural Representation/ Pattern}

Since the 3D structure of each metalloprotein has been converted into the respective $1 \mathrm{D}$ letter sequence as described above, the letters that correspond to the metalbound amino acid residues yielded a structural represen- tation of the first-shell, as shown in the last columns of Additional files 1 and 3 for each metal-binding site. For example, in the case of the human/chicken estrogen receptor (1HCQ), the letters corresponding to the $\mathrm{Zn}$ binding Cys residues at position 7, 10, 24 and 27 are $f, o$, $f$, and $m$, respectively, yielding a $f(2) o(13) f(2) m$ representation of the first-shell for 1HCQ (see Figure 1).

\section{Definition of Structural Motifs}

In previous work [29], all values of $k$ between 2 and 20 were used to define a structural motif, where $k$ is the number of first-shell structural patterns with the same structural letters and similar interletter spacing. Here, $k \geq$ 3 was used to define a structural motif. Thus, if 3 or more proteins possess first-shell structural patterns with the same structural letters and similar interletter spacing, these proteins are assumed to share a common structural motif. For example, transketolase (1ITZ), pyruvate oxidase (1POX), 2 oxo-acid dehydrogenase alpha subunit (1UMD), pyruvate decarboxylase (1ZPD), and pyruvateferredoxin oxidoreductase $(2 \mathrm{C} 3 \mathrm{M})$ share the first-shell structural pattern, $k(26-29) h(1) a$, which thus defines a structural motif.

\section{Authors' contributions}

MD carried out all the calculations, including writing programs, and drafted the manuscript. CL conceived of the study, participated in its design and analysis/interpretation of data, and writing/revising the manuscript. Both authors have read and approved the final manuscript. 


\section{Additional material}

\section{Additional file 1}

The $M g^{2+-d a t a s e t ~ c o n t a i n i n g ~} 77$ metal-binding sites in 70 nonredundant $\mathrm{Mg}^{2+-}$ proteins. A table listing the PDB entries, protein description, native metal-cofactors (if known), EC code, metal-bound amino acid residues, and first-shell structural representation of the 70 nonredundant $\mathrm{Mg}^{2+-}$ proteins.

Click here for file

[http://www.biomedcentral.com/content/supplementary/14712105-8-106-S1.doc]

\section{Additional file 2}

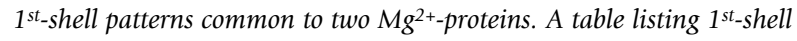
structural patterns that is common to only $2 \mathrm{Mg}^{2+-b i n d i n g ~ s i t e s . ~}$

Click here for file

[http://www.biomedcentral.com/content/supplementary/1471-

2105-8-106-S2.doc]

\section{Additional file 3}

The $\mathrm{Ca}^{2+}$-dataset containing 230 metal-binding sites in 177 nonredundant $\mathrm{Ca}^{2+}$-proteins. A table listing the PDB entries, protein description, native metal-cofactors (if known), EC code, metal-bound amino acid residues, and first-shell structural representation of the 177 nonredundant Ca $a^{2+}$-proteins.

Click here for file

[http://www.biomedcentral.com/content/supplementary/14712105-8-106-S3.doc]

\section{Acknowledgements}

We thank anonymous reviewers for constructive comments/suggestions. We are grateful to Steven Wu, Michael J. B. Lin, and Backy Chen for assistance in the statistical analyses, and Leon Li, Todor Dudev, and Gopi Kuppuraj for literature assistance. This work was supported by NSC contract no. NSC 94-21 I3-M-001-018 to CL.

\section{References}

I. Cowan JA: Biological Chemistry of Magnesium. New York, $\mathrm{VCH} ; 1995$.

2. Nordlund $\mathrm{P}$, Sjoberg BM, Eklund $\mathrm{H}$ : Three-dimensional structure of the free radical protein of ribonucleotide reductase. Nature 1990, 345:593.

3. Dudev T, Cowan JA, Lim C: Competitive Binding in Magnesium Coordination Chemistry: Water versus Ligands of Biological Interest. J Am Chem Soc 1999, I 2 1:7665-7673.

4. Cowan JA: Metal activation of enzymes in nucleic acid biochemistry. Chem Rev 1998, 98:1067-1087.

5. Chotia C, Lesk AM: The relation between the divergence of sequence and structure in proteins. $E M B O J$ 1986, 5:823-826.

6. de Brevern AG, Etchebest C, Hazout S: Bayesian probabilistic approach for predicting backbone structures in terms of protein blocks. Proteins: Struct Funct Genet 2000, 41:27I-287.

7. de Brevern AG: New assessment of a structural alphabet. In Silico Biol 2005, 5:26.

8. Unger R, Sussman JL: The importance of short structural motifs in protein structure analysis. I Comput Aided Mol Des 1993, 7:457-472.

9. Tyagi M, Sharma P, Swamy CS, Cadet F, Srinivasan N, de Brevern AG, Offman B: Protein block expert (PBE): a web-based protein structure analysis server using a structural alphabet. Nucleic Acids Res 2006, 34:WII9-WI23.

10. Tyagi M, Gowri VS, Srinivasan N, de Brevern AG, Offmann B: A substitution matrix for structural alphabet based on structural alignment of homologous proteins and its applications. Proteins: Structure, Function \& Bioinformatics 2006, 65(I):32-39.

II. Bystroff C, Baker D: Prediction of local structure in proteins using a library of sequence- structure motifs. J Mol Biol 1998, 28 I (3):565-577.

12. Kolodny R, Koehl P, Guibas L, Levitt M: Small libraries of protein fragments model native structures accurately. J Mol Biol 2002, 323:297-307.

13. Fourrier L, Benros C, de Brevern AG: Use of a structural alphabet for analysis of short loops connecvting repetitive structures. BMC Bioinformatics 2004, 5:58.

14. Dudev T, Lin YL, Dudev M, Lim C: First-Second Shell Interactions in Metal Binding Sites in Proteins: A PDB Survey and DFT/CDM Calculations. J Am Chem Soc 2003, I 25:3 I68-3 I80.

15. Petkovich M, Brand NJ, Krust A, Chambon P: A human retinoic acid receptor which belongs to the family of nuclear receptors. Nature 1987, 330:444-450.

16. Berman HM, Battistuz T, Bhat TN, Bluhm WF, Bourne PE, Burkhardt K, lype L, Jain S, Fagan P, Marvin J, Padilla D, Ravichandran V, Schneider B, Thanki N, Weissig H, Westbrook JD, Zardecki C: The Protein Data Bank. Acta Crystallogr D 2002, 58:899-907.

17. Sigrist CJ, Cerutti L, Hulo N, Gattiker A, Falquet L, Pagni M, Bairoch A, Bucher P: PROSITE: a documented database using patters and profiles as motif desriptors. Brief Bioinform 2002, 3:265-274.

18. Dudev T, Lim C: Principles Governing Mg, Ca, and Zn Binding and Selectivity in Proteins. Chem Rev 2003, 103:773-787.

19. Lahiri SD, Zhang GF, Dunaway-Mariano D, Allen KN: Diversification of function in the haloacid dehalogenase enzyme superfamily: The role of the cap domain in hydrolytic phosphorussingle bondcarbon bond cleavage. Bioinorg Chem 2006, 34:394-409.

20. Iding H, Dunnwald T, Greiner L, Liese A, Muller M, Siegert P, Grotzinger J, Demir AS, Pohl M: Benzoylformate decarboxylase from Pseudomonas putida as stable catalyst for the synthesis of chiral 2-hydroxy ketones. Chemistry -A Eur J 2000, 6: 1 483-I 495.

21. Watson JD, Laskowski RA, Thornton JM: Predicting protein function from sequence and structural data. Curr Op Struct Biol 2005, 15:275-284.

22. Kristensen DM, Chen BY, Fofanov VY, Ward RM, Lisewski AM, Kimmel M, Kavraki L, Lichtarge O: Recurrent use of evolutionary importance for functional annotation of proteins based on local structural similarity. Prot Sci 2006, I5:1530-1536.

23. Mathura VS, Schein $\mathrm{CH}$, Braun W: Identifying property based sequence motifs in protein families and superfamilies: application to DNase-I related endonucleases. Proteins: Structure, Function and Bioinformatics 2003, 19:138I-1390.

24. Schein CH, Zhou B, Oezguen N, Mathura VS, Braun W: Molegobased definition of the architecture and specificity of metalbinding sites. Proteins: Structure, Function and Bioinformatics 2005, 58:200-210.

25. Laskowski RA: PDBsum: summaries and analyses of PDB structures. Nucleic Acids Res 200I, 29(I):22I-222.

26. Allen $\mathrm{FH}$ : The Cambridge structural database: a quarter of a million crystal structures and rising. Acta Cryst 2002, B58:380-388.

27. Harding MM: The geometry of metal-ligand interactions relevant to proteins. Acta Cryst 1999, D55:1432-1443.

28. McDonald IK, Thornton JM: Satisfying hydrogen bonding potential in proteins. J Mol Biol 1994, 238(5):777-793.

29. Jonassen I, Eidhammer I, Conklin D, Taylor WR: Structure motif discovery and mining the PDB. Bioinformatics 200I, 1 8:362-367. 